\title{
Trajectory Arrangement of Bucket Motion of Wheel Loader
}

\author{
SARATA Shigeru (AIST)*, OSUMI Hisashi(Chuo University), \\ HIRAI Yusuke(Canon Co.) and MATSUSHIMA Gen (Citizen Co.) \\ * Intelligent Systems Laboratory, \\ National Institute of Advanced Industrial Science and Technology \\ Namiki 1-2-1, Tsukuba, 305-8564 JAPAN \\ sarata-s@aist.go.jp
}

\begin{abstract}
For autonomous scooping operation by wheel loader, a method for adequate arrangement of bucket trajectory will be proposed. In scooping operation of wheel loader by human operator, bucket motion includes several cycles of arm lift and bucket tilt alternately. Consequently, the tip of bucket moves on alternate short path of horizontal and vertical direction. In this paper, relation between resistance force and advancing direction is analyzed theoretically. Advancing direction of the bucket is dominant factor for resistance force on the bucket during scooping. Based on this analysis, simple algorithm for bucket trajectory arrangement is proposed. The algorithm is implemented into small size experimental model of wheel loader. Results of the experiments show that proposed algorithm generates adequate bucket motion for different conditions of pile.
\end{abstract}

KEYWORDS: Wheel Loader, Scooping, Bucket Control, Trajectory, Resistance force, Pile

\section{INTRODUCTION}

A wheel loader (front-end loader) is one of the major loading machines in construction work, mining, agriculture etc. For automatic or autonomous loading operation, basic studies have been carried out and several methods for bucket trajectory generation have been proposed. One of major method is similar to the computed torque method, which is applied for control of serial link manipulators used in assembling lines[1]. In this method, the reference value for the control system is the estimation value of resistance force at the bucket based on soil-bucket interaction models. However, soil-bucket interaction models are based on estimated value of unknown factors such as bulk density, angle of internal friction etc. These factors cannot be obtained prior to scooping.

By contrast, other methods, which do not use any information on the pile or material, have been applied in field trials and shown good performance. In the scoop operation by human operator, scoop procedure consists of several cycles of arm lift and bucket tilt alternately. To realize this motion, a kind of sequence controller was installed and applied to scooping operation at asphalt plant and gravel mine[2][3]. The controller performed this cyclic bucket motion based on the resistance force at the bucket.
In ordinary scooping by wheel loader, bucket motion consists of three phases as shown in Fig.1. At the beginning of scooping, the bucket is placed on the ground. The base of bucket is kept horizontally. The bucket penetrates into the pile with advancing of the body by wheel revolution in Phase 1(A-B). In Phase 2(B-C), the tip of bucket moves upward along a line or a curve with increasing of the tilt angle of bucket. When the bucket is filled up with the pile, the bucket moves upward almost vertically in Phase 3(C-D). Determination of transfer from Phase 1 to Phase 2 and arrangement of bucket path are significant for effective scooping.

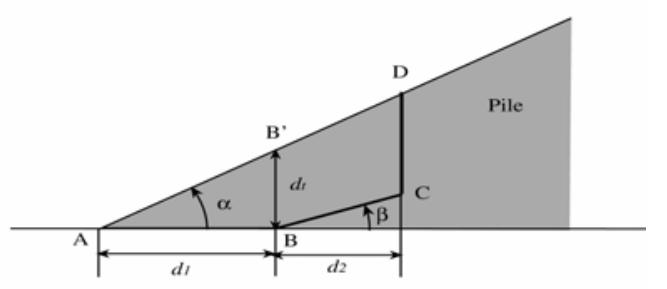

Figure 1. Bucket Path Model

In operation by human operator, it seems that when penetrating force exceeds "threshold", Phase is transferred from 1 to 2. In Phase 2, Arm lift and tilt up are repeated cyclically. Arm lift generates 
mainly forward movement of the bucket. Tilt up generates upward movement.

In this paper, these empirical operation methods is analyzed theoretically and evaluated by experiment using small size experimental model of wheel loader.

\section{ELEMENTS OF FORCE ACTING ON THE BUCKET}

Fig. 2 shows geometrical parameters of the bucket motion and pile at scooping. Each elements of reacting force applied on the bucket are shown in Fig.3. Definition and formulations of each element are as follows;

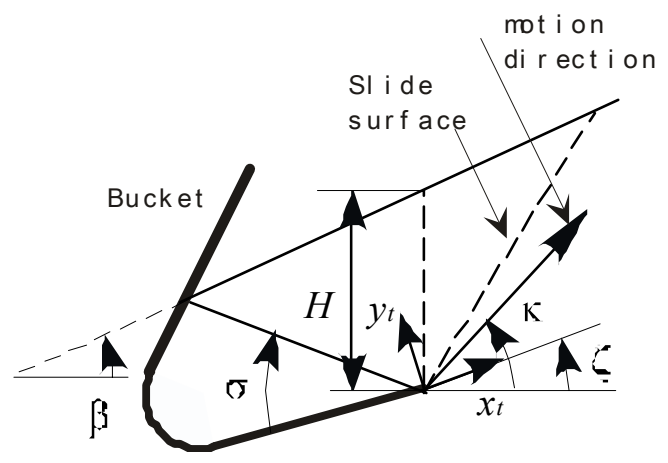

Figure 2. Geometrical Parameters

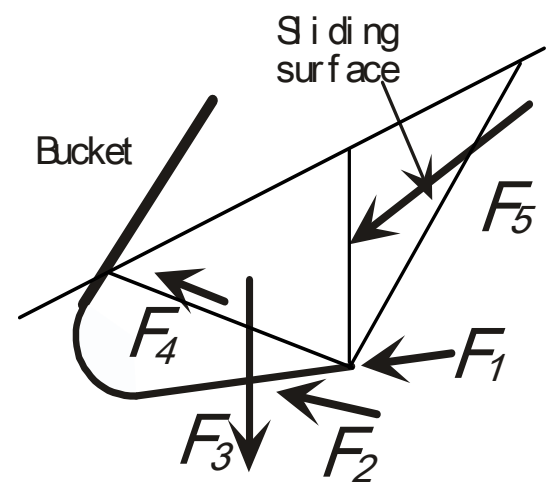

Figure 3. Force Acting on Bucket

$F_{l}$ : penetrating force at the tip of bucket during advancing.

$F_{1}=K_{0} \cos \kappa \gamma H S g$

Where $H$ is depth of pile at the tip of bucket, $S$ is cross sectional area of the tip of bucket, $\gamma$ is specific gravity of material of the pile and $g$ is acceleration of gravity. $K_{0}$ is coefficient related penetration resistance for each material.
$F_{2}$ : Resistance force at bottom of the bucket. $F_{2}$ appear when $\kappa>\zeta$ in Figure 2 however such direction or configuration are not taken at practice operation.

$F_{3}$ : weight of material in the bucket.

$F_{3}=M g$

where $M$ is mass of material in the bucket.

$F_{4}$ : Frictional force between bucket and materials inside of the bucket. Vertical elements of force applied on inside of the bucket include that of $F_{5}$.

$F_{4}=\mu F_{3} \cos \zeta+\mu F_{5} \cos (\phi-\zeta)$

$F_{5}$ : Force required to move material in front of the bucket. $F_{5}$ varies depend on scooping phases. In phase 2, Material inside of the bucket forms virtual plane that push material outside of the bucket. In this case, passive soil pressure by Coulomb theory can be applied with consideration the virtual plane as the wall.

$$
P_{P}=\frac{\gamma H^{2}}{2} \frac{1}{\sin ^{2} \alpha \sin (\alpha-\delta)} \times
$$

$$
\frac{\sin ^{2}(\phi+\alpha)}{\left[1+\sqrt{\frac{\sin (\phi+\delta) \sin (\phi-\beta)}{\sin (\alpha-\delta) \sin (\alpha+\beta)}}\right]^{2}}
$$

For

formularization of elements of the force, basic experiments have been carried out. Fig. 4 shows experiment system. The main component of the system is slide table driven by boll screw and DC motor. Experimental plates are attached on an end of the table and resistance force is measured during penetration into pile.

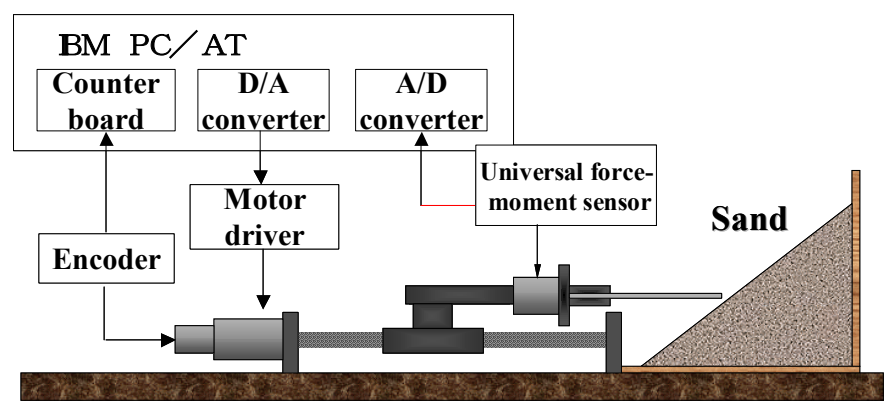

Figure 4. Penetration Force Measurement 
Fig.6 is the result of flat plane penetration as shown in Fig.5(a). Width of the plates is $150 \mathrm{~mm}$ and thickness of plates are $2 \mathrm{~mm}$ and $3 \mathrm{~mm}$. Dark line and gray line represent resistance force of $3 \mathrm{~mm}$ and $2 \mathrm{~mm}$ plate respectively. Thin line represents calculated frictional force obtained by extrapolation. It is clear that penetrating force F1 is proportional to depth of pile and frictional force is proportional to power of the depth. Magnitude of the penetrating force is superior to the frictional force.

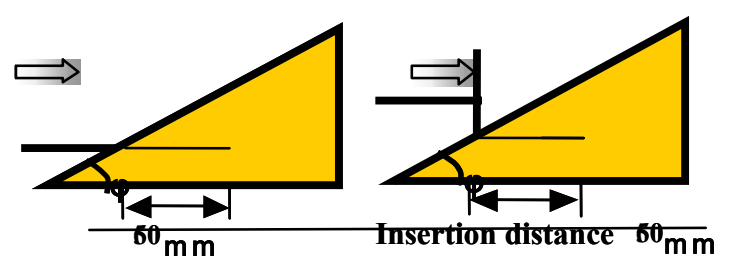

(a) flat plate

(b) vertical plate

Figure 5. Types of Plate

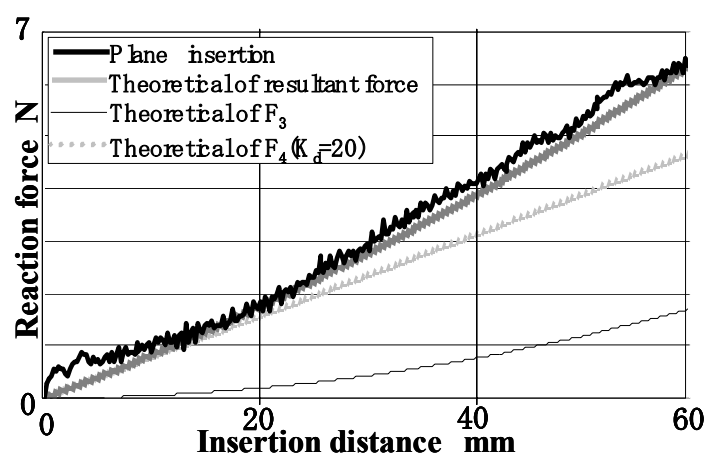

Figure 6. Resistance Force at Flat plate

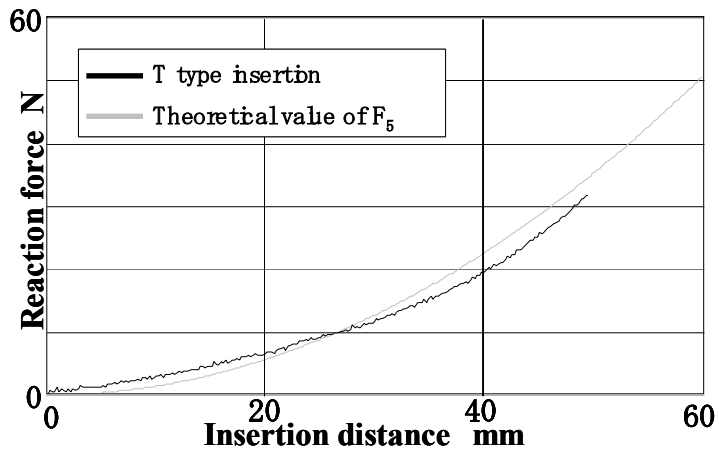

Figure 7. Resistance Force at Vertical plate

Fig.7 is the result of vertical plate penetration as shown in Fig.5(b). Thin line represents calculation result of resistance force based on Coulomb soil pressure theory. Both of lines agree very well. Formulation of $F_{5}$ can be based on Coulomb's theory. The result shows that the magnitude of resistance force in this case is superior to those of the penetrating force or the frictional force. $F_{5}$ is dominant force in resistance force at the bucket.

\section{KINEMATICS AND FORCE ANALYSIS OF BUCKET LINK}

In this section, kinematics and force analysis of the experimental model YAMAZUMI-2(YZ-2 Fig.8) is described. Fig.9 shows kinematics model of YZ-2. The bucket motion mechanism consists of two links: arm link and tilt link. The bucket has three degrees of freedom with linear body motion by wheel revolution. Therefore position and configuration of the bucket are represented by functions of three valuables: advancing of the body, arm angle and tilt angle.

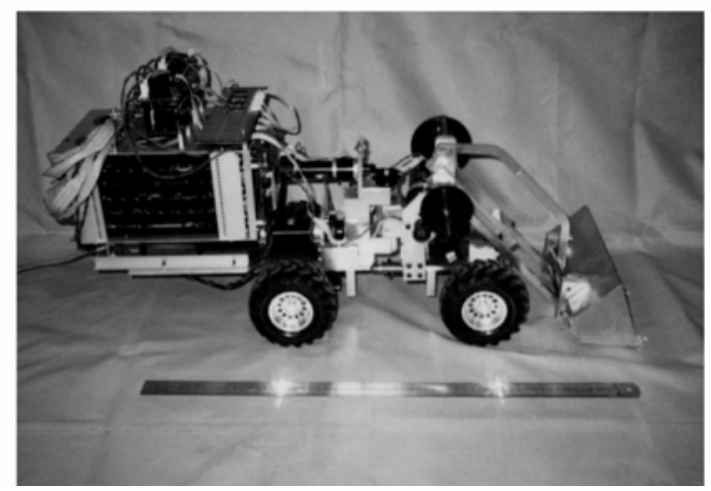

Figure 8. YAMAZUMI-2

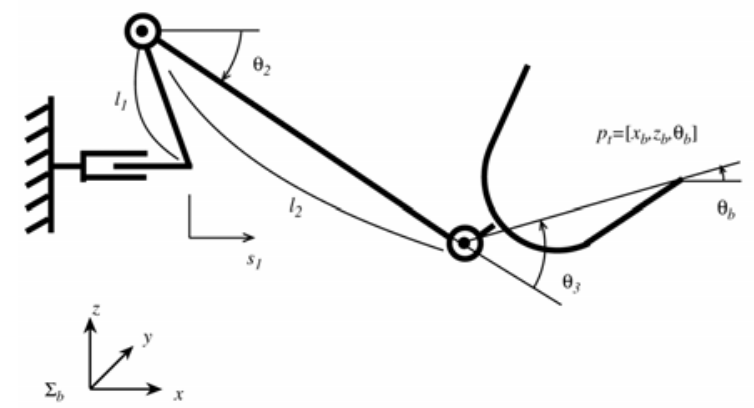

Figure 9. Kinematics Model

$$
\begin{aligned}
& p_{x}=q_{1}+l_{1}+l_{3} \cos \left(q_{2}\right)+l_{4} \cos \left(q_{3}+\theta_{b}\right) \\
& p_{y}=l_{2}+l_{3} \sin \left(q_{2}\right)+l_{4} \sin \left(q_{3}+\theta_{b}\right) \\
& \theta_{p}=q_{3}+\theta
\end{aligned}
$$


where $\boldsymbol{p}=\left(p_{x}, p_{y}, \theta_{p}\right)^{T}$ is the position vector of bucket tip, $\boldsymbol{q}=\left(q_{1}, q_{2}, q_{3}\right)^{T}$ is the joint vector, $q_{1}$ is the advancing distance, $q_{2}$ is the angle of arm and $q_{3}$ is the angle of bucket tilt. $\theta_{b}$ is the offset angle between direction of $l_{4}$ and the bucket.

Matrix equation (6) is obtained by differentiating above equations. This equation describes relation between joint velocity and bucket velocity.

$$
\dot{\boldsymbol{p}}=\boldsymbol{J} \dot{\boldsymbol{q}}
$$

Where $\dot{\boldsymbol{p}}$ is bucket velocity vector, $\dot{\boldsymbol{q}}$ is joint velocity vector and $\boldsymbol{J}$ is Jacobian matrix.

Relation between force at the bucket and torque at each joint is described by the equation.

$$
\tau=J^{T} f
$$

Where $\boldsymbol{J}^{T}$ is transposed matrix of Jacobian. $\tau=\left(\tau_{1}, \tau_{2}, \tau_{3}\right)^{T}$ is the torque vector and each element is the torque at each joints respectively. $\boldsymbol{f}=\left(f_{x}, f_{y}\right.$, $\left.n_{x y}\right)^{T}$ is the external force vector. Operational force at the tip of bucket is obtained from equation (8) with inverse matrix of $\boldsymbol{J}^{T}$.

$$
f=\left(J^{T}\right)^{-1} \tau
$$

$f_{x}$ and $f_{y}$ are obtained from equation (8),

$$
\begin{aligned}
& f_{x}=\tau_{1} \\
& f_{y}=\tau_{1} \tan \theta_{2}+\tau_{2} / l_{2} \cos \theta_{2}
\end{aligned}
$$

Let $\tau_{1 \max }$ and $\tau_{2 \max }$ be the maximum torque at wheel and arm actuators. The maximum operational force at the tip of bucket is shown in Fig. 10 at a certain configuration. Each point in the polygon of operational force represents combination of $f_{x}$ and $f_{y}$ which are element of $x$ and $y$ direction of the force at the tip of bucket as described in equation (9).

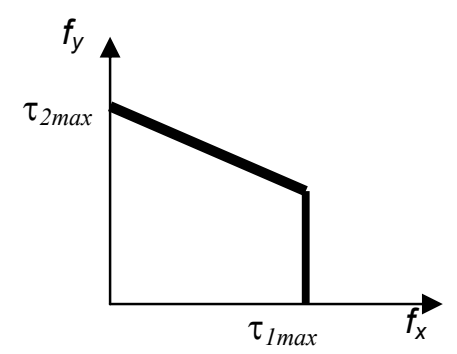

Figure 10. Operational Force Plygon

\section{RESISTANCE FORCE ENVELOPE AND OPERATIONAL FORCE}

As described in previous sections, magnitude of the resistance force $F_{1}, F_{4}$ and $F_{5}$ at the bucket from pile is varied with advancing direction of the bucket. However $F_{3}$ has no relation to the advancing direction. Apparent resistance force on the bucket is integrated force from $F_{1}$ through $F_{5}$. In fig.11, magnitude of $F_{l}$ in arbitrary advancing directions is shown. In this figure, the tip of bucket is located on the origin of this coordinate system. When the bucket moves in direction $\boldsymbol{p}$, magnitude of penetration force is length of vector p.

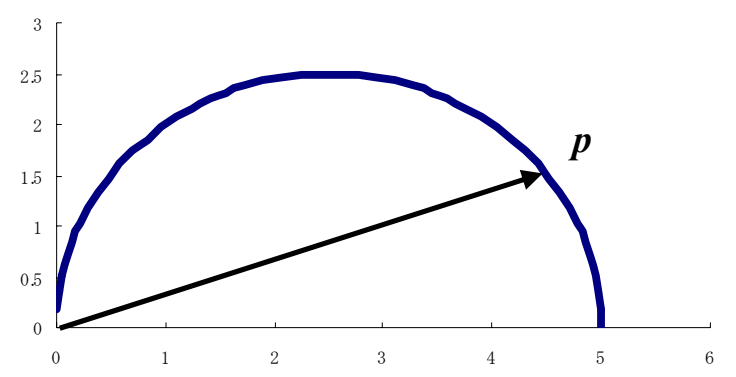

Figure 11. Resistance Force $F_{1}$

$F_{4}$ and $F_{5}$ have close relation to each other. As shown in Fig.12, virtual plane is supposed between materials inside and outside of the bucket. $F_{5}$ acts in normal direction to this virtual plane.

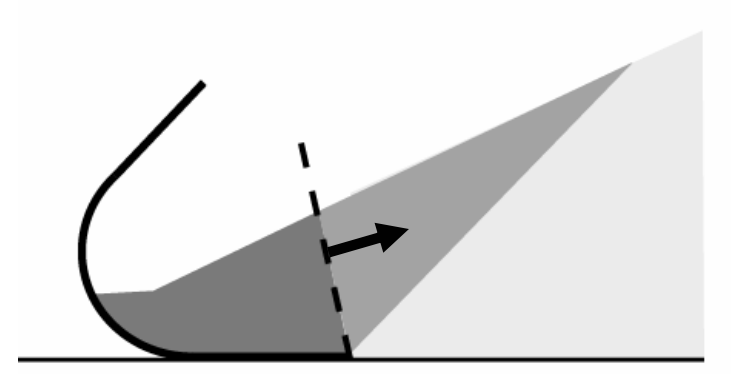

Figure 12. Virtual Plane

When advancing of the bucket, it pushes material on the virtual plane. Required force for the bucket movement is greater than that of $F_{5} . F_{5}$ is formularized by passive pressure of Coulomb theory. Direction of the virtual plane is normal to advancing direction of the bucket. Therefore magnitude of $F_{5}$ is function of the advancing 
direction. On the virtual plane, no shear stress is supposed to be appeared. Hence $F_{5}$ is obtained by equation (4) with $\delta=0$. Fig.13 shows magnitude of $F_{5}$ in each advancing directions.

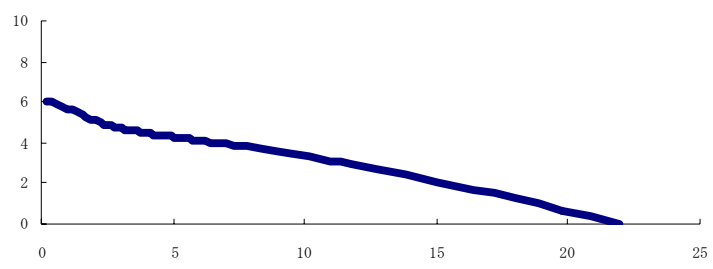

Figure 13. Magnitude of $F_{5}$

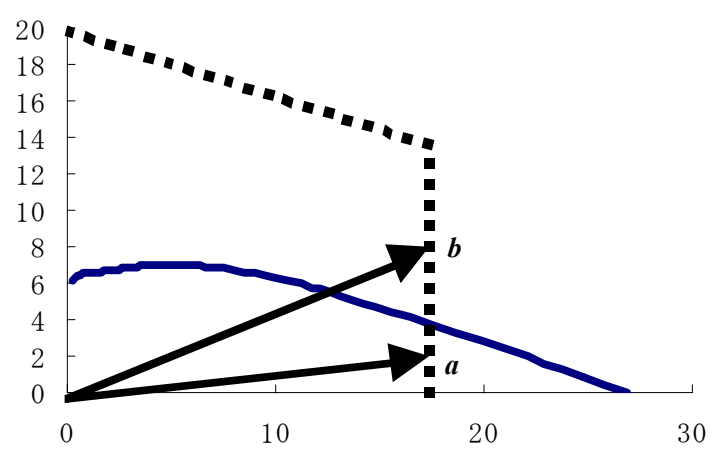

Figure 14. Resistance Force Envelope and Operational Force Polygon

Integration of $F_{1}$ and $F_{5}$ form resistance force envelop as shown in Fig.14. It is total required force for advancing of the bucket in corresponding directions. Dotted lines represent operational force polygon. If the bucket moves indirection $\boldsymbol{a}$, magnitude of resistance force exceeds operational force. Therefore the bucket cannot move in this direction. If the bucket moves in direction $\boldsymbol{b}$, operational force is greater than resistance force and the bucket can move in this direction. Direction for intersection of resistance force envelope and operational force polygon is lower limit of advancing direction.

By advancing of the bucket, resistance force is increased proportional to increasing of depth of the pile at the tip of bucket. Then the bucket cannot move in this direction because the resistance force exceeds the operational force in this direction. However the bucket can continue advancing in upper direction because the resistance force is smaller in these directions.

\section{TRAJECTORY ARRANGEMENT}

Based on analysis described in previous sections, an algorithm for bucket control is installed on experimental model YM-2. The algorithm consists of simple rule: if horizontal element of resistance force exceed setting threshold, advancing direction of the bucket is arranged to upward direction. Arranged angle is proportional to exceeding value of the resistance force from the threshold.

Bucket of YZ-2 is $250 \mathrm{~mm}$ in width, and has capacity of $2000 \mathrm{~cm}^{3}$. Experimental pile is crashed granite in $5 \mathrm{~mm}$ diameter. Specific gravity is 1.4 and internal frictional angle is 38 degree.

Fig.15 (a) and (b) show path of the tip of bucket. Inclined lines are slope of the pile. Slope angles of pile are (a) 20degree and (b) 30degree. Setting threshold for controller is $20 \mathrm{~N}$. In both of the cases, path of tip shows that scooping is separated into phases defined in the beginning section. Bucket motion is smooth and continuous. The proposed control algorithm generates adequate motion of the bucket for different conditions of pile.

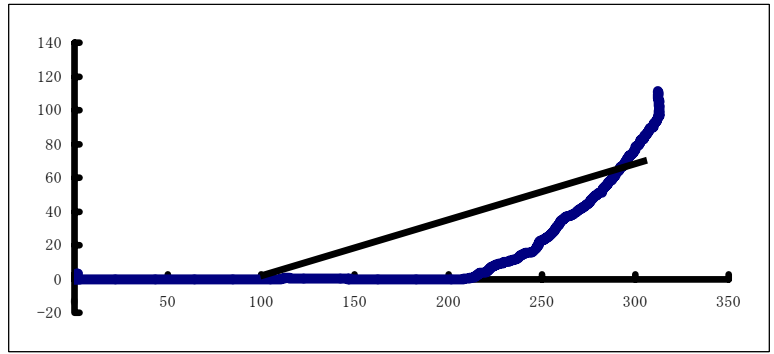

(a) Gentle slope(20degree)

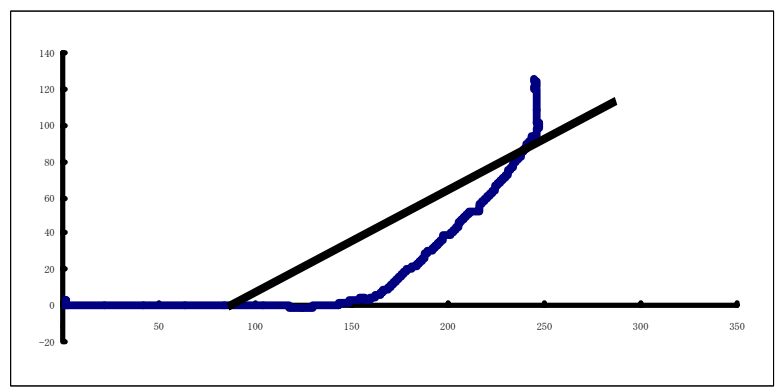

(b) Steep slope(30degree)

Figure 15. Path of Tip of Bucket

In phase 1, the bucket moves into pile horizontally and penetration length are $110 \mathrm{~mm}$ in (a) and $50 \mathrm{~mm}$ in (b). At these points, depth of pile on the 
tip of bucket is $40 \mathrm{~mm}$ in (a) and $30 \mathrm{~mm}$ in (b). Relation between resistance force and height of the virtual plane obtained by equation (4) is shows in Fig.16. X-axis represents height of virtual plane in $[\mathrm{mm}]$ and $y$-axis represents resistance force in $[\mathrm{N}]$. Dark line represents case (a) and gray line represents case (b). If resistance force is $20 \mathrm{~N}$, corresponding height of virtual plane is $40 \mathrm{~mm}$ and $30 \mathrm{~mm}$ for case (a) and (b) respectively. These depths agree with experimental results.

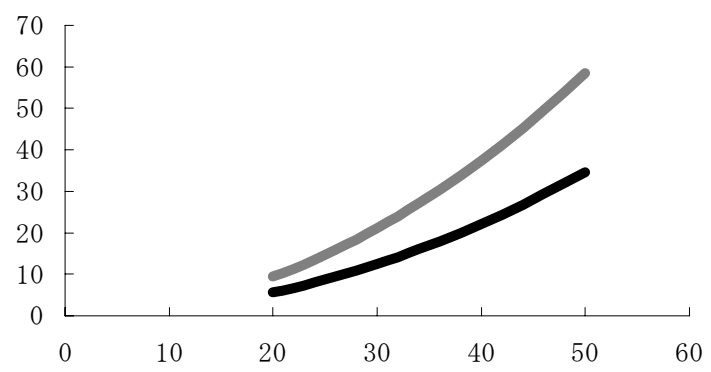

Figure 16. Resistance force vs. height of the virtual plane

At the beginning of Phase 2, the bucket moves in upper direction in (b) than (a). Fig.17 shows relation between resistance force and advancing direction of bucket. Magnitude of the resistance force depends on direction significantly. Dark line represents case (a) and gray line represents case (b). If advancing direction is changed upward by 10 degrees, resistance force is reduced to half.

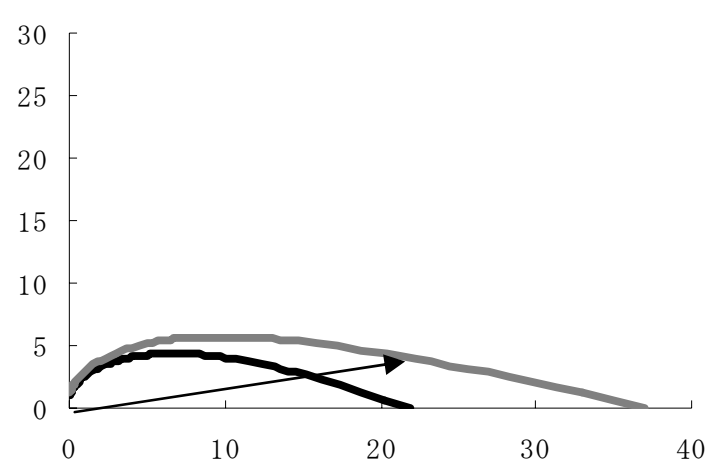

Fig.17 Resistance Force

\section{CONCLUSION}

Relation between the resistance force and advancing direction of bucket is analyzed theoretically. The relation is formularized in the resistance force envelope and the maximum operational force of bucket motion mechanism. The advancing direction is dominant factor for resistance force on the bucket. Based on the analysis, simple algorithm for bucket trajectory arrangement is implemented on experimental model YZ-2. The result shows that the proposed algorithm generates adequate bucket motion for different condition of pile. The proposed algorithm shows good performance for different conditions in experiments however combination with bucket trajectory planning with vision system would be one of the practical solutions for application to real working sites..

\section{REFERENCES}

[1]Singh,S. and Cannon,H.:Milt-Resolution Planning for Earthmoving, Proc ICRA, 1998

[2]Gocho,T. et al.: Autonomous Wheel-loader in Asphalt Plant, Proc. 9th International Symposium on Automation and Robotics in Construction, 1992

[3]Sarata,S.: Research and Development on Unmanned Loading Operation by Wheel Loader, Proc. Rapid Mine Development, 2002 